\title{
Mise en évidence d'un facteur stimulant la sécrétion de prolactine et de l'hormone de croissance dans les drèches de brasserie
}

\author{
L. Sawadogo, H. Sepehri et L. M. Houdebine
}

Laboratoire de biologie cellulaire et moléculaire, Unité de différenciation cellulaire, INRA, 78350 Jouy-en-Josas, France

(reçu le 13-7-1988, accepté le 28-11-1988)

Résumé - Des extraits aqueux de drèches de brasserie injectés par voie intraveineuse à des brebis ou à des vaches induisent la sécrétion de prolactine et d'hormone de croissance (GH). Les mêmes drèches, ajoutées à la ration alimentaire de vache, se sont révélées incapables de stimuler de manière significative le taux de prolactine et de $\mathrm{GH}$ dans le sang. Dans ces conditions expérimentales, la production laitière n'était pas augmentée. Le B-glucane pur extrait d'orge stimule également la sécrétion des hormones lorsqu'il est administré par voie intraveineuse. L'orge, la bière et les drèches de brasserie contiennent donc un facteur de type B-glucane qui stimule la sécrétion des hormones lactogènes. Le contenu des drèches en facteur est probablement insuffisant pour provoquer une augmentation des hormones lorsqu'elles sont administrées par voie orale. L'effet stimulant classique des drèches de brasserie sur la production laitière doit donc être dû à leur apport nutritif plutôt qu'à une intervention du système endocrinien.

drèches - prolactine - hormone de croissance - lactation

Summary - Presence of a stimulatory factor of prolactin and growth hormone secretion in brewery draff. Aquous extracts of brewery draff injected intravenously into ewes and cows induced prolactin and growth hormone (GH) secretion. The same draff added to the feed of cows appeared to be unable to significantly stimulate the blood level of prolactin and $\mathrm{GH}$. In these experimental conditions, milk production was not enhanced by draff. Pure B-glucan extracted from barley also stimulated hormone secretion when administered by the intravenous route. Barley, bier and draff therefore contain a B-glucan-like factor which stimulates lactogenic hormone secretion. The amount present in draff is probably unable to cause an increase in hormones when administered orally. Hence, the well-established stimulatory effect of draff on milk production results from their nutritive value rather than from their ability of modulating the endocrine system.

draff - prolactin - growth hormone - lactation 


\section{Introduction}

Les drèches de brasserie sont classiquement utilisées comme nourriture pour les ruminants. Elles sont réputées augmenter de manière significative la production laitière (Arnould et al., 1983). Le mécanisme par lequel cette stimulation est exercée n'a pas été défini avec certitude bien que les apports nutritifs des drèches pourraient rendre compte de leur pouvoir galactogène.

La bière est connue pour stimuler la sécrétion lactée des femmes allaitantes. Une étude récente a démontré que la bière contient effectivement un facteur qui, administré par voie orale, est capable d'induire la synthèse de caséine dans les glandes mammaires de rate et de stimuler la sécrétion de prolactine et d'hormone de croissance (Sawadogo et Houdebine, 1988b). Le facteur est soluble dans l'eau et insoluble dans les solvants organiques. II provient du malt et de l'orge mais non du houblon. La fraction active obtenue à partir de l'orge ou du malt est très riche en B-glucane (Sawadogo et al., 1988b).

Les expériences qui sont décrites ici ont été entreprises pour déterminer si les drèches de brasserie contiennent également le facteur galactogène trouvé dans la bière et le malt, et si leur pouvoir galactogène est tout ou partie le résultat d'une augmentation de la sécrétion de prolactine et d'hormone de croissance.

\section{Matériels et Méthodes}

\section{Extraction du facteur}

Des drèches fraîches ont été chauffées dans l'eau bouillante pendant $15 \mathrm{~min}$. La partie aqueuse lyophilisée est considérée comme l'extrait total; il représente environ $2 \%$ du poids initial de drèches. La partie aqueuse a égale- ment été agitée en présence d'un demi-volume de chloroforme et centrifugée. La partie aqueuse a été recueillie et le facteur a été insolubilisé par l'addition de 2 volumes d'éthanol. La fraction insolubilisée a été collectée par centrifugation à basse vitesse et lyophilisée. Elle représente $0,5 \%$ du poids de drèches initial. Cette méthode est essentiellement celle décrite pour l'extraction du facteur lactogène présent dans le malt (Sawadogo et Houdebine, 1988b).

Mesure de la sécrétion de prolactine et de $G H$

Les fractions extraites après dissolution dans du sérum physiologique des drèches ont été injectées par voie intraveineuse à des brebis ou des vaches. Des échantillons de sang ont été prélevés toutes les $10 \mathrm{~min}$ avant et après l'injection des extraits. Les hormones ont été dosées dans le plasma par des tests radioimmunologiques spécifiques permettant de déceler $1 \mathrm{ng}$ d'hormone par $\mathrm{ml}$ de plasma. Ces méthodes sont exactement celles décrites dans des publications récentes (Sawadogo, 1987; Sawadogo et Houdebine, 1988a).

Le B-glucane pur d'orge (Sigma) a également été dissous dans le sérum physiologique. La partie insoluble a été éliminée par centrifugation avant l'injection.

Mesure de l'effet des drèches administrées par voie orale

Deux lots de 14 vaches traites depuis 4 mois ont reçu $15 \mathrm{~kg}$ de drèches par jour à $9 \mathrm{~h} 30 \mathrm{le}$ matin. La mesure de la production laitière et la collecte d'échantillons sanguins ont commencé 4 jours avant le début du traitement. Les prélèvements de sang ont été faits à $9 \mathrm{~h}, 11 \mathrm{~h} 15$ et $16 \mathrm{~h}$, soit avant et après l'administration de drèches.

\section{Résultats}

Action des extraits de drèches administrées par voie intraveineuse

Des extraits totaux de drèches injectés par voie intraveineuse à des brebis stimu- 
lent de manière intense la sécrétion de prolactine (Fig. 1). Cette stimulation est semblable pour l'essentiel à celle obtenue avec les extraits de bière, d'orge, de malt (Sawadogo et Houdebine, 1988b) ou d'autres plantes ayant également un pouvoir galactogène (Sawadogo et Houdebine, 1988a; Sawadogo et al., 1988a et b).

Des extraits plus purifiés sont également capables de stimuler la sécrétion de prolactine et de $\mathrm{GH}$ chez la Brebis et chez la Vache lorsqu'ils sont administrés par voie intraveineuse (Fig. 2). Les drèches de brasserie contiennent donc un facteur galactogène. Le fait qu'il soit extrait par l'eau et insolubilisé par l'éthanol suggère que ce facteur est le même que celui identifié dans la bière et le malt (Sawadogo et Houdebine, 1988b).

\section{Action du B-glucane d'orge}

Une analyse chimique de la composition de la fraction active extraite du malt a révélé que le B-glucane était la molécule dominante tandis que les extraits des autres plantes galactogènes étaient tous riches en pectines (Sawadogo et al., 1988b). Les pectines et certains de leurs dérivés se sont révélées posséder les mêmes propriétés que les extraits de plantes (Sawadogo et al., 1988a), ce qui suggère que la molécule active dans les extraits de ces plantes est l'acide polygalacturonique. I paraissait donc logique de déterminer l'activité de polymère de B-glucane pur extrait d'orge. Les résultats de la Figure 3 indiquent sans ambiguité que cette macromolécule est capable de stimuler très franchement la sécrétion de prolactine et de $\mathrm{GH}$ chez des brebis lorsqu'elle est administrée par voie intraveineuse. Il est donc tentant de conclure que le facteur galactogène présent dans l'orge, le malt, la bière et les drèches de brasserie est un polymère de B-glucane.

Action des drèches administrées par voie orale

Des quantités substantielles de drèches (15 kg par jour et par animal) ont été données à des vaches en cours de lactation. Le lot témoin a reçu une nourriture conte-
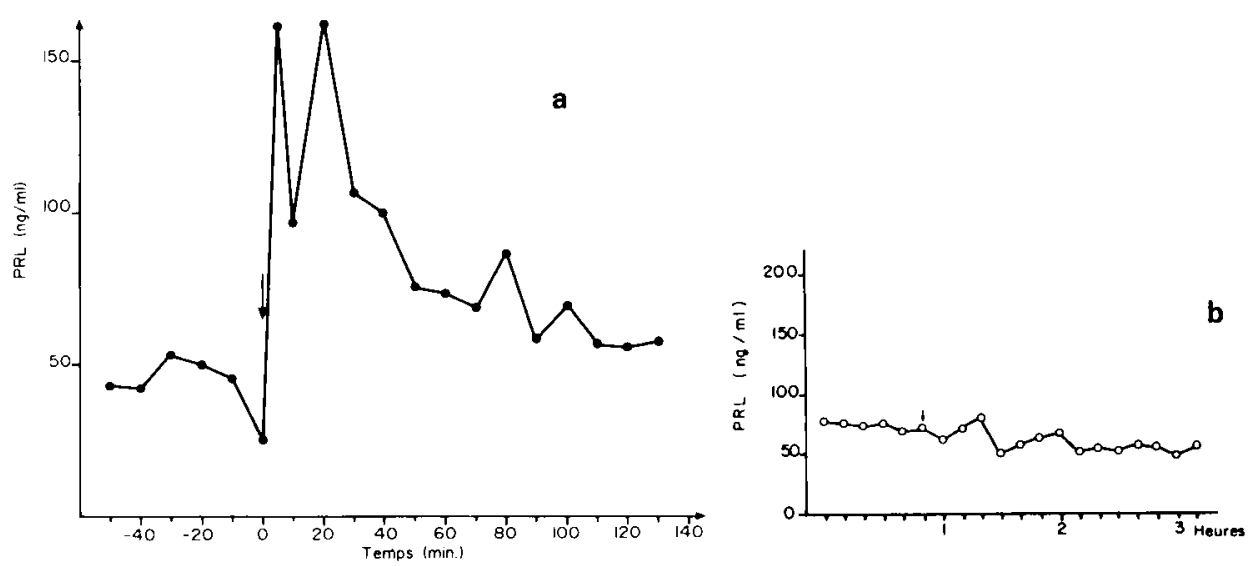

Fig. 1. Effet d'un extrait total de drèches sur la sécrétion de prolactine. A. L'extrait $(500 \mathrm{mg})$ a été dissous dans du sérum physiologique $(5 \mathrm{ml})$ et injecté par voie intraveineuse à une brebis au moment indiqué par la flèche. B. Injection de sérum physiologique. 

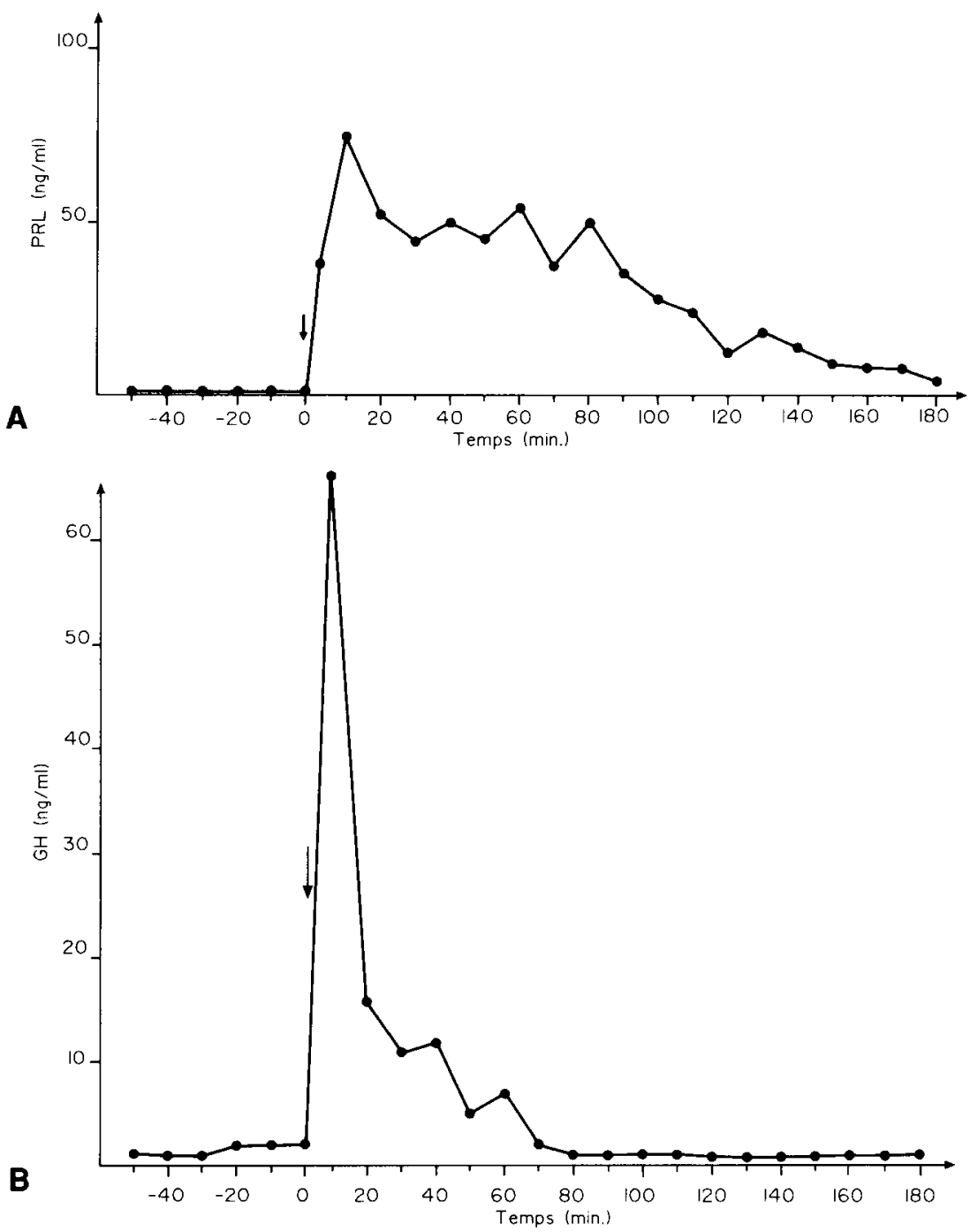

Fig. 2. Effet d'un extrait purifié de drèches sur la sécrétion de prolactine et de GH. L'extrait $(1 \mathrm{~g})$ a été dissous dans du sérum physiologique $(5 \mathrm{ml})$ et injecté à une vache par voie intraveineuse au moment indiqué par la flèche. A. Niveau sanguin de prolactine. B. Niveau sanguin de GH.

nant la même quantité d'énergie et d'azote, de manière à ce que les drèches représentent une substitution dans la ration alimentaire et non un supplément.

Les résultats résumés dans la Figure 4 indiquent que les drèches administrées dans ces conditions n'altèrent pas de manière significative la sécrétion de prolactine et de GH. La seule différence, qui n'est que de faible amplitude, est observée dans les échantillons prélevés à $9 \mathrm{~h}$ le matin. 


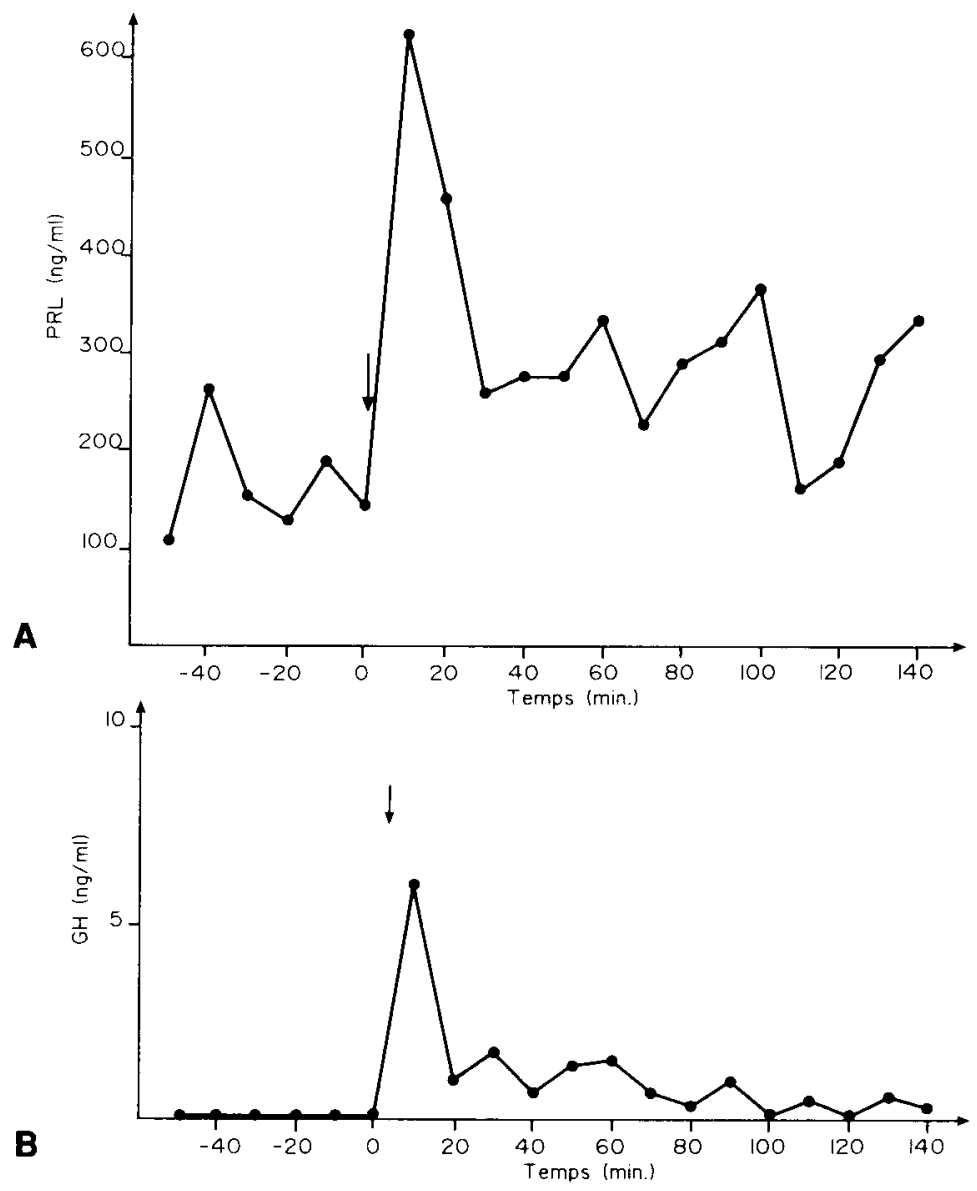

Fig. 3. Effet du B-glucane sur la sécrétion de prolactine et de GH. Le B-glucane (100 $\mathrm{mg})$ a été dissous dans du sérum physiologique et injecté (après centrifugation) à une brebis par voie intraveineuse au moment indiqué par la flèche. A. Niveau sanguin de prolactine. B. Niveau sanguin de GH.

Dans ces conditions expérimentales, les drèches n'ont pas modifié la production laitière de manière significative (Fig. 5).

\section{Conclusion}

Les expériences décrites ici indiquent que les drèches de brasserie comme la bière, l'orge et le malt contiennent un facteur capable de stimuler la sécrétion de prolactine et de $\mathrm{GH}$. Chez la rate, ce facteur est capable de stimuler la sécrétion des hormones lorsqu'il est administré par voie orale et il induit en même temps une synthèse de lait, ce qui démontre son véritable pouvoir lactogène (Sawadogo et Houdebine, 1988a; Sawadogo et al., 1988a). Chez la Brebis et la Vache, les mêmes extraits sont capables de stimuler 

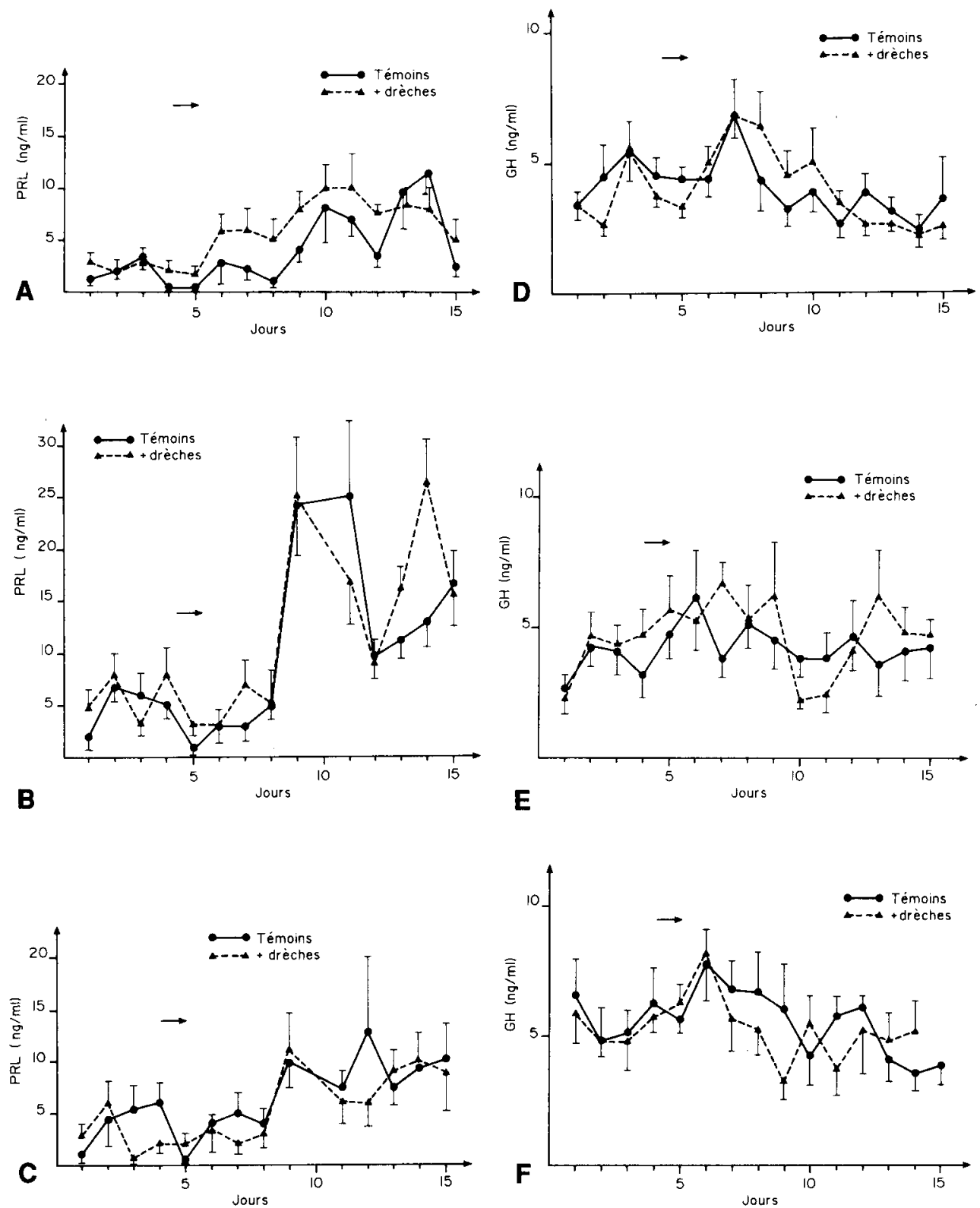

Fig. 4. Action des drèches sur la sécrétion de prolactine et de $\mathrm{GH}$ après administration orale. Deux lots de 14 vaches ont reçu ou non des drèches $(15 \mathrm{~kg})$ à $9 \mathrm{~h} 30$ le matin (à partir du jour indiqué par la flèche). Des prélèvements de sang ont été faits à $9 \mathrm{~h} 00$ (A et $D$ ), $11 \mathrm{~h} 15$ (B et $E$ ) et $16 \mathrm{~h} 00$ ( $C$ et F). A, B, C. Niveau sanguin de prolactine. D, E, F. Niveau sanguin de GH. $(\bullet-\bullet)$ témoins; $(\Lambda)+$ drèches. Les valeurs sont la moyenne des 14 animaux ( \pm SEM) pour la prolactine et de 5 animaux pris au hasard $( \pm$ SEM) pour la GH. 


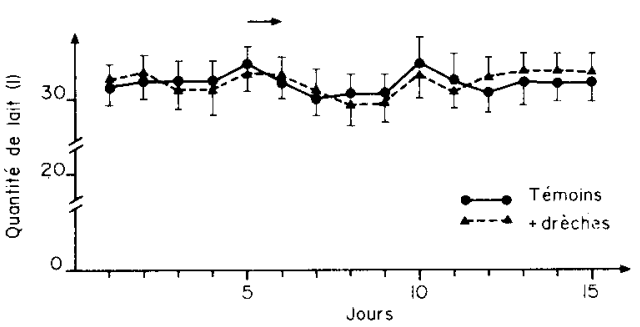

Fig. 5. Production laitière moyenne des vaches après administration de drèches. Les animaux sont ceux décrits à la Figure 4. (-) témoins; ( $1-\downarrow)+$ drèches. La flèche indique le début de l'administration de drèches.

la sécrétion des hormones à condition d'être administrés par voie intraveineuse. Ce mode d'administration se traduit par un apport massif et rapide du facteur dans la circulation sanguine. Par voie orale, le facteur ne doit atteindre le sang que relativement lentement, ce qui peut expliquer son incapacité à stimuler la sécrétion des hormones. $\mathrm{Ce}$ facteur est toutefois actif par voie orale chez la rate. Le long transit que doit subir le facteur avant de pouvoir pénétrer dans le sang peut correspondre à un effet de dilution qui se traduit par un effet négligeable ou nul sur la sécrétion d'hormone. II est également concevable que le facteur est rapidement inactif dans le tractus digestif des polygastriques et non dans celui des monogastriques. II est également possible que le facteur soit présent en trop petite quantité dans les drèches. En effet, le facteur étant hydrosoluble, la majeure partie de ce qui se trouvait initialement dans l'orge doit se retrouver dans la bière et non dans les drèches. L'apport des drèches en facteur peut donc être de toute façon insuffisant pour avoir un effet stimulant franc.

Il est possible que les conditions d'utilisation des drèches dans les expériences décrites ici ne soient pas les meilleures puisque la production laitière n'a pas subi d'augmentation significative.

Quoi qu'il en soit, il y a peu de chance que l'effet traditionnellement observé des drèches de brasserie sur la production laitière des animaux domestiques soit le résultat d'une stimulation du système endocrinien.

Un point reste intriguant : les extraits de malt et de B-glucane d'orge stimulent la sécrétion de 2 hormones au moins. A notre connaissance, ce fait n'avait pas été rapporté jusqu'à ce jour. La même constatation avait été faite en ce qui concerne les extraits des autres plantes galactogènes, des pectines et de l'acide polygalacturonique (Sawadogo et al., 1988a). L'acide polygalacturonique n'est actif qu'à l'état de polymère. II est frappant de rapprocher ces observations de celles faites pour les plantes. Les polymères de l'acide galacturonique et de B-glucane sont considérés comme des hormones ou des messagers hormonaux chez les plantes (Ryan, 1987). Les résultats décrits ici et dans une autre publication (Sawadogo et al., 1988a) suggèrent de manière inattendue que les cellules animales sont également sensibles à ces messagers hormonaux. Ces observations posent la question de savoir quels sont les récepteurs sensibles aux 2 polysaccharides dans les cellules animales, quel est leur mode d'action et quelles molécules du règne animal sont normalement reconnues par ces récepteurs.

\section{Références}

Arnould R., Bouquiaux J.M. \& Vanbelle M. (1983) L'utilisation des drèches de brasserie (malt et houblon) dans l'alimentation des vaches laitières. Rev. Agric. (Bruss.) 1, 93-105 Ryan C.A. (1987) Oligosaccharide signalling in plants. Annu. Rev. Cell. Biol. 3, 295-317 
Sawadogo L. (1987) Contribution a l'étude des plantes médicinales et de la pharmacopée traditionnelle africaine : cas des plantes lactogènes. Thèse de Doct. ès Sci. Tours

Sawadogo L. \& Houdebine L.M. (1988a) Induction de la synthèse de caséine- $\beta$ dans la glande mammaire de rates traitées par des extraits de plantes. C.R. Acad. Sci. Paris 306, 167-172

Sawadogo L., Houdebine L.M., Thibault J.F., Rouaux X. \& Ollivier-Bousquet M. (1988a)
Effect of pectic substances on prolactin and growth hormone secretion in the ewe and on the induction of casein synthesis in the rat. Reprod. Nutr. Dev. 28, 293-301

Sawadogo L. \& Houdebine L.M. (1988b) Identification of the lactogenic compound present in beer. Ann. Biol. Clin. 46, 129-134

Sawadogo L., Houdebine L.M., Thibault J.F. \& Rouaux X. (1988b) Mise en évidence d'une activité lactogène dans des extraits végétaux. Bull. Med. Trad. 2, 19-30 